\title{
EDITORIAL
}

\section{Design Creativity: Mechanism, Tools and Applications}

\author{
Thanh An Nguyen* \\ Department of Electrical and Computer Engineering, Concordia University, Montreal, Canada
}

In the 1950 presidential address to the American Psychological Association, Guilford (1950) emphasized the importance of creativity research and urged researchers to adopt scientific approaches to the topic. Today, creativity has become one of the most popular and significant research topics, crossing disciplinary boundaries. This issue consists of five papers exploring creativity from different perspectives. The first two papers study creativity related problems from cognitive perspective; the next two papers focus on development of creativity support tools and the last paper explores the link between creativity and humour.

Divergent thinking has long been associated with creativity. The paper titled "When does a paper clip become a sundial? Exploring the progression of originality in the alternative uses test" aims to understand how the Alternative Uses Test relates to originality. The Alternative Uses Test is a divergent thinking test in which the testees are asked to list unusual uses for an ordinary object. In the experiment, the authors asked 293 participants to list alternative uses of a paper clip as many as they can in three minutes. The contribution of the paper is twofold. First, the analysis shows that participants who generate more ideas have more original ideas and the first nine responses are less likely to be original. Based on this result, the authors suggest that experimenters should not limit the examinees' responses to 6 items for the Alternative Uses Test as in its current version. Responders should be required to produce at least 9 ideas to ensure the originality of the idea. The same advice goes to a brainstorming meeting where the chair should encourage participants to think of at least 9 responses. Second, from the analysis of the test, the authors create a database that can be used in scoring the digital format of the Alternative Uses Test in real time.

The second paper titled "Considering confirmation bias in design and design research" studies confirmation bias, a cognitive factor that obscures creativity. In the paper, the authors explore the prevalence of confirmation bias in design and design research. Confirmation bias is a tendency to validate beliefs rather than invalidate them. Confirmation bias is one of the factors leading to failures of adopting appropriate design solutions because designers tend to accept a design that they believe. Designers justify their choice by relying on supporting evidence and underrating, or even overlooking, conflicting evidence. Confirmation bias can also influence design researchers. To investigate this phenomenon, the authors asked sixteen researchers to evaluate a hypothesis using given data. The participants are divided into two groups: one group uses decision matrix to evaluate the data and the other group does not use any method. The result reveals that most of the subjects in the intuition group (no method) confirms that the hypothesis is true and their evaluation is bias towards confirming evidence. In contrast, the matrix group shows similar responses to the ideal answers. From this study, the authors address the possible impact of confirmation bias on scientific research and review methods to mitigate the effect.

\footnotetext{
* Corresponding author. Email: ngu_th@encs.concordia.ca. Tel: (+1)514-8482424 ext 7043.
} 
"When people talked about innovation in the '90s, they really meant technology. When people talk about innovation in this decade, they really mean design" (Nussbaum, 2005). That statement emphasizes the tie between design and creativity in this century. A design process can be seen as a process of creating an artifact and assigning a meaning to the artifact within its environment (Zeng, 2011). Therefore, creativity in design is the process of assigning a surprisingly new meaning to an artifact. The third paper titled "Linguistic readymades and creative reuse" proposes an architecture to help users retrieve potential readymades (clichés, quotes or phrases with cultural resonance) to be re-used in a new context. The reuse is a creative act because the new context will render new meaning to the readymades. The architecture, called creative Information Retrieval (creative IR), pulls readymade candidates from a variety of sources such as corpora, Wikipedia and N-gram database. Besides the conventional wildcard widely used in search engine, the module includes additional wildcards such as pragmatic neighbourhood wildcard, cultural stereotype wildcard, antonym wildcard, ad-hoc category wildcard and the combination of these. Although the creative IR does not aim to become a cognitive model of human creativity, it can serve as one of the components in the model of future machine creativity.

To make strategic decision, companies need to forecast innovative trend. The fourth paper titled "Integrated model for technology assessment and expected evolution: a case study in the Chilean mining industry" proposed a systematic approach to analysing technological advancement. Despite of a handful of techniques available for technology assessment and forecasting, these methods are labour-intensive and lacks temporal information. Therefore, the aim of the paper is to introduce a new method that overcomes these issues. The proposed method combines TRIZ and patent analysis to forecast the next technological evolution and the time it is to be realized. Two case studies in Chilean mining industry are illustrated to show the advantages and limitations of the method.

Finally, the last paper titled "Humour and creative design: twins or partners" investigates the relationships between humour and creative design process. First, the authors argue that humour and creative design are twins because they share a similar mechanism. Specifically, the expectation of the story ending is framed by the beginning and the current script. As readers read, the reader expectations are framed and updated. This resembles a design process in which user requirements are clarified and revised along the design process. Furthermore, humour effect and creative design solution are both based on the relationship between expectations and reality. Second, the authors reason that humour and creative design are also partners because they can be interdependent. Humorous mood stimulates design creativity and the process of creating humour implies a creative design process.

Despite of recent growth in research activities, our understanding of creativity is still limited. In the next few years, research in creativity will continue to bring us with new exciting findings and applications.

\section{References}

Guilford, J. P. (1950). Creativity. American Psychologist, 5(9), 444-454. doi: http://dx.doi.org/10.1037/h0063487

Nussbaum, B. (2005). Getting Schooled in Innovation, Bloomberg BusinessWeek. Retrieved from http://www.businessweek.com/stories/2005-01-02/getting-schooled-in-innovation

Zeng, Y. (2011). Environment-Based Design (EBD). ASME Conference Proceedings, 2011(54860), 237250.

\section{Author Biography}

Thanh An Nguyen is a Ph.D. student in Electrical and Computer Engineering Department at Concordia Unversity, Montreal, Canada. She received her Bachelor's degree in Computer Science and Master's degree in Information System Security, both from Concordia University. Her research interests include design cognition, human visual perception and curve reconstruction. 